\title{
MaNGA: Mapping Nearby Galaxies at Apache Point Observatory
}

\begin{abstract}
Kevin Bundy
Kavli Institute for the Physics and Mathematics of the Universe, Todai Institutes for Advanced Study, the University of Tokyo, Kashiwa, Japan 277-8583 (Kavli IPMU, WPI)

Abstract. I describe a new integral field spectroscopic survey called MaNGA (Mapping Nearby Galaxies at Apache Point Observatory). One of three core programs in the 6-year SDSS-IV project $\dagger$ that began on July 1st, 2014, MaNGA will deploy 17 fiber-bundle IFUs across the Sloan $2.5 \mathrm{~m}$ Telescope's 3 degree field-of-view, targeting a mass-selected sample with a median redshift of 0.03 , typical spatial resolution of $1-2 \mathrm{kpc}$, and a per-fiber signal-to-noise ratio of 4-8 in the outskirts of target galaxies. For each galaxy in the sample, MaNGA will provide maps and measured gradients of the composition and dynamics of both stars and gas. I discuss early results that highlight MaNGA's potential to shed light on the ionization and chemical enrichment of gas in galaxies, spatial patterns in their star formation histories, and the internal makeup of stellar populations. MaNGA's unprecedented data set will not only provide powerful new insight on galaxy formation and evolution but will serve as a valuable benchmark for future high-z observations from large telescopes and space-based facilities.
\end{abstract}

Keywords. galaxies: evolution - galaxies: formation

\section{Introduction}

Our understanding of galaxy formation has significantly advanced in the last decade thanks to SDSS surveys at low redshift, a variety of higher redshift surveys and observations, and improvements in simulating non-linear structure formation. While at the present day the largest dark matter halos continue to grow from the "bottom-up" by merging with smaller halos, the galaxies living in these halos experienced peak star formation rates at $z \sim 1-3$. From this time on, activity steadily declined while galaxies continued to grow via mergers and star formation fueled by the accretion of fresh gas. Eventually, star formation was somehow shut down. This quenching process, a kind of "galaxy death," increasingly affects lower mass systems with time and may soon be the fate of our own Milky Way. Present-day galaxies less massive than the Milky Way formed their stars continuously over all cosmic epochs. In fact, the majority of the stars in galactic disks formed in the last 7-9 billion years.

In spite of significant progress in delineating this basic picture, important pieces of the puzzle remain to be understood. Very importantly, we still seek the physical origin of the patterns of evolution described above. Gravitational collapse is only one of many processes at work in the formation of a galaxy. Gas dissipation and infall, star formation, heating and ejection of gas by supernova explosions, galaxy merging, the redistribution of material due to internal processes, and associated growth of central black holes are all key processes that remain poorly understood.

One limitation has been the fact that a single spectroscopic measurement at a galaxy's center cannot capture the rich physics responsible for its internal structure and dynamics. Two-dimensional, integral-field spectroscopy (IFS) is needed and recent efforts such as

$\dagger$ www.sdss.org 
SAURON (de Zeeuw et al. 2002), DiskMass (Bershady et al. 2010), ATLAS ${ }^{3 \mathrm{D}}$ (Cappellari et al. 2011), and CALIFA (Sánchez et al. 2012) have demonstrated enormous promise, although their small $($ few $\times 100)$ and incomplete samples hamper statistical studies. The more recently launched SAMI survey (utilizing the Sydney Australian Astronomical Observatory Multi-object Integral Field Spectrograph, Croom et al. 2012) is making major gains using a multiplexed fiber-IFU instrument. MaNGA represents another advance, surveying 10,000 galaxies across a wide dynamic range in $M_{*}$, environment, and star formation rate with uniform radial coverage. Especially important is MaNGA's unique wavelength coverage in the near-IR, the location of diagnostics sensitive to the IMF, such as $\mathrm{NaI}$ and the CaII triplet (Conroy \& van Dokkum 2012), as well as important tracers of thermally-pulsating asymptotic giant branch (TP-AGB) stars and other intermediateage populations (Maraston 2005). Combined with its sensitivity in the near-UV, MaNGA will open up simultaneous access to a large number of compositional features and dynamic tracers. A comprehensive overview of the MaNGA project is given in Bundy et al. (2014).

\section{Instrument and Survey Design}

The MaNGA instrument couples a novel technology that delivers regular, high-throughput fiber integral field units (IFUs) with the sensitive spectrographs. The instrument and the target selection have been designed and optimized in concert.

MaNGA IFUs: MaNGA's high-performance fiber bundles have "fill factors" of 56\%. To uniformly cover targets in order to reconstruct their light distribution requires requires dithered observations and highly uniform fiber spacing. Through the development of breakthrough technology (see below), MaNGA achieves a packing regularity that is $1-2 \%(3 \mu \mathrm{m})$ from the ideal hexagonal configuration. Careful attention to fiber stress, length, and application of anti-reflective coating allows MaNGA to reach lab-measured 95\% throughput across all fibers in our bundles. Our fabrication technology scales easily to yield a range of IFU sizes from 19 to 127 fibers with identical performance optimized for our galaxy sample.

Spectroscopy: MaNGA makes use of the high-sensitivity BOSS spectrographs (Smee et al. 2013), each of which features a red and blue arm for continuous wavelength coverage from the near-UV into the near-IR. MaNGA packs 1423 fibers into the spectrographs, enabling input from 17 science IFU bundles, twelve 7-fiber flux calibration bundles, and 92 sky fibers. With a typical resolution of $R \sim 2000$, MaNGA samples a broad wavelength region, including the region of $750 \mathrm{~nm}<\lambda<1000 \mathrm{~nm}$. This expansive and continuous coverage captures a large range of absorption features, from blue indices such as D4000, $\mathrm{Ca} \mathrm{H}+\mathrm{K}$, and higher-order Balmer lines, through classical optical absorption features such as $\mathrm{H} \beta, \mathrm{Mg} b$, and $\mathrm{Fe} 5270 / \mathrm{Fe} 5335$, to red, gravity-sensitive features such as the $\mathrm{Ca}$ triplet at $860 \mathrm{~nm}$. These features encode a great deal of information about star formation histories and the IMF, and can be used to measure element abundance ratios such as $[\mathrm{Mg} / \mathrm{Fe}],[\mathrm{N} / \mathrm{Fe}],[\mathrm{C} / \mathrm{Fe}]$ and $[\mathrm{Ca} / \mathrm{Fe}]$. The spectrograph's sensitivity $(\epsilon)$ and solid angle $(\Omega)$ combined with the Sloan $2.5 \mathrm{~m}$ Telescope's (Gunn et al. 2006) collecting area (A) yield an unparalleled etendue $(\mathrm{A} \Omega \epsilon)$.

Sample Optimization: Maximizing S/N and spatial resolution demands target galaxies with the lowest possible redshifts. Typical ground-based sampling of the radial extent of small galaxies with a least three resolution elements (assuming FWHM $\sim 2.0$ ") requires $z \lesssim 0.03$. However, building large samples with representative numbers of high-mass galaxies requires large volumes and therefore demands redshifts $z>0.03$. At a median redshift of $\sim 0.03$, as a result of the cosmic galaxy number density, a sweet spot exists to 


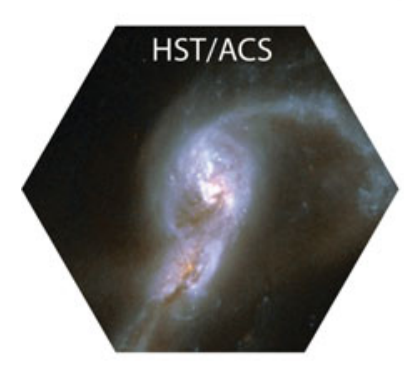

\section{Mrk 848: SDSS-IV/MaNGA First-Article Data Cube}
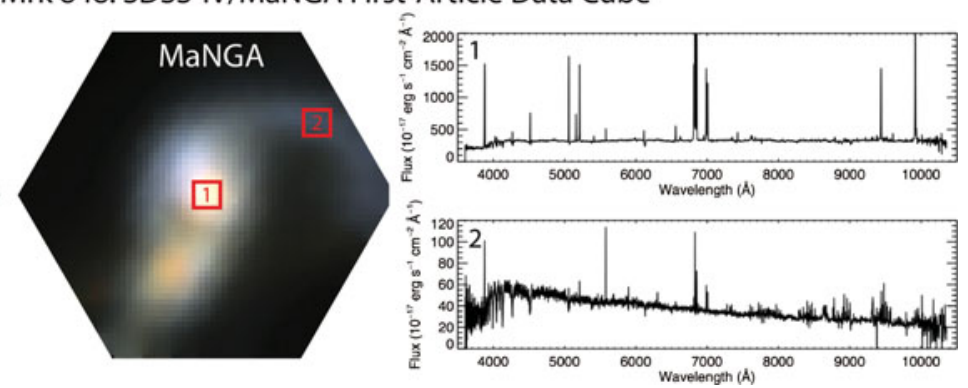

Figure 1. Color HST image of Markarian 848 compared to the reconstructed MaNGA color image for the same galaxy. MaNGA spectra from two different regions are also plotted. Credit: David Law.

gather a sample size of many thousand of galaxies in a survey covering several thousand $\mathrm{deg}^{2}$. The sky density of such a sample is $3 \mathrm{deg}^{-2}$, and fortuitously is well matched to the MaNGA IFU complement made possible by Sloan's $7 \mathrm{deg}^{2}$ field of view and the BOSS spectrographs.

We have performed an exhaustive optimization of IFU bundle size distribution, yielding 17 IFUs with between 19 and 127 fibers that ensure radial coverage to $1.5 R_{\mathrm{e}}$ and, for gradient measures requiring the highest $\mathrm{S} / \mathrm{N}\left(\gtrsim 30 \AA^{-1}\right)$ via annular binning, a minimum of three resolved annuli (e.g., $\Delta r=2 "$ ). On average, MaNGA galaxies are covered by five annular bins that result in sufficient stacked $\mathrm{S} / \mathrm{N}$ to derive robust stellar population parameters (e.g., age, metallicity, element abundances). For $\mathrm{S} / \mathrm{N}=8$, dithered MaNGA observations provide over 80 unique, radial samples for the average target galaxy (the median MaNGA physical spatial resolution is $1-2 \mathrm{kpc}$ ). The sample is volume-limited as a function of $i$-band luminosity so that roughly equal numbers of the lowest $\left(10^{9} \mathrm{M}_{\odot}\right)$ and highest mass $\left(10^{11.5} \mathrm{M}_{\odot}\right)$ galaxies are obtained. We intend to devote $1 / 3$ of the full sample to an exploratory study of the physical properties at $2.5 R_{\mathrm{e}}$, a regime never yet probed with significant statistics. MaNGA targets are selected solely from the SDSS-I MAIN sample and are adaptively tiled to probe the full range of central and satellite galaxies in all halos $\left(\log \left(M_{h} / M_{\odot}\right)=10.5-15\right)$ and local density environments.

\section{Early Results}

Three studies based on data obtained in 2013 using a prototype of the MaNGA instrument have now been submitted for publication. Belfiore et al. study maps of the ionization states of gas in this P-MaNGA sample. Wilkinson et al. develop a new spectral fitter to derive maps of the age, metallicity, dust, and surface mass density of the stellar populations. Li et al. map diagnostics of recent star formation like D4000 and H $\delta$ to explore constraints on recent fueling and quenching.

Commissioning of the final MaNGA instrumentation began in March 2014 and the first data cubes have been produced. The galaxy, Markarian 848, was targeted on the first MaNGA plate and is shown in Figure 1. A more typical MaNGA galaxy is shown in Figure 2.

\section{Acknowledgements}

SDSS is managed by the Astrophysical Research Consortium for the Participating Institutions. These include Carnegie Mellon University, Colorado University, Boulder, Harvard- 

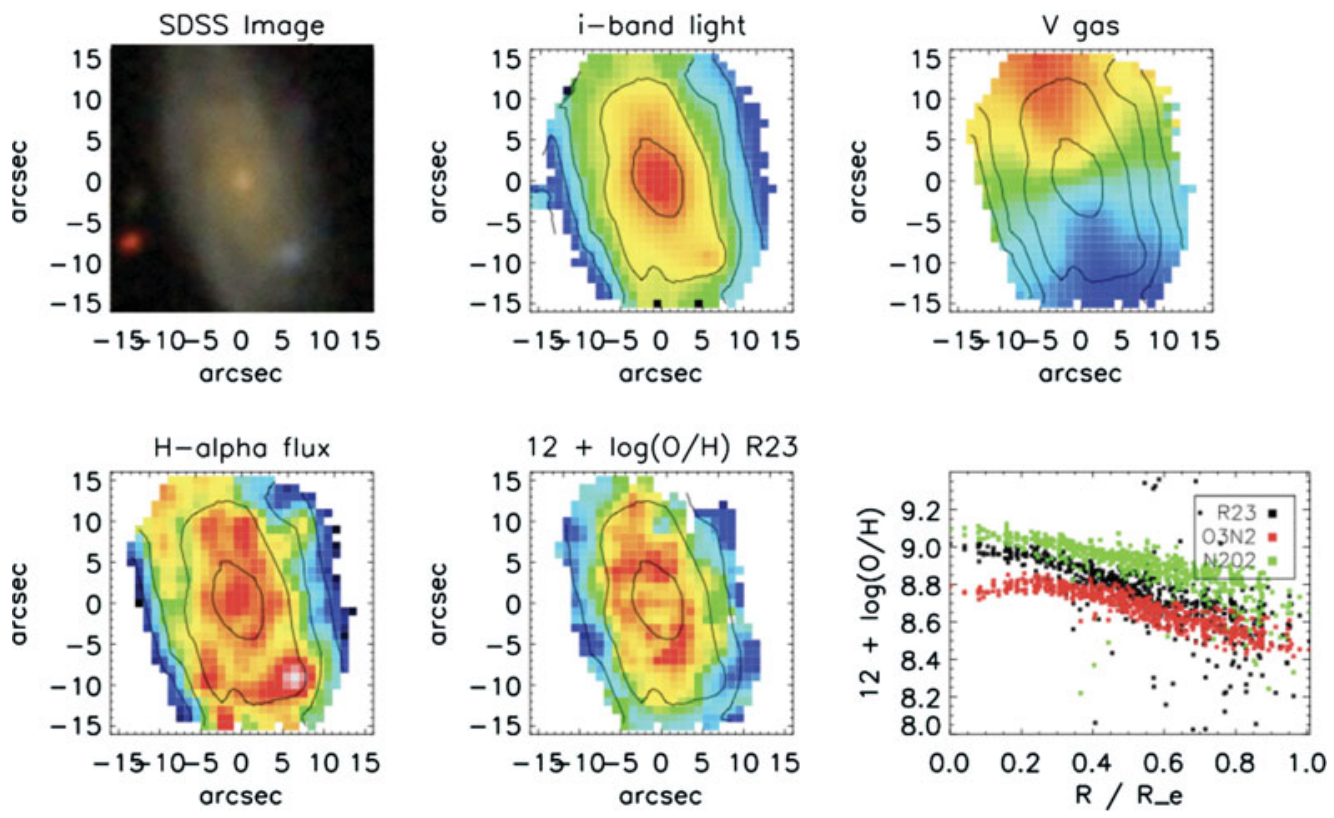

Figure 2. A typical star-forming galaxy as observed during MaNGA commissioning in March 2014. Maps of the $i$-band flux, gas velocity (as measured from $\mathrm{H} \alpha$ ), $\mathrm{H} \alpha$ flux, and R23 gas-phase metallicity are shown. The bottom right panel plots gas-phase metallicity gradients using three popular indicators. Individual points represent separate spaxels in the data cube for this galaxy. Credit: Christy Tremonti.

Smithsonian Center for Astrophysics Participation Group, Johns Hopkins University, Kavli Institute for the Physics and Mathematics of the Universe Max-Planck-Institut fuer Astrophysik (MPA Garching), Max-Planck-Institut fuer Extraterrestrische Physik (MPE), Max-Planck-Institut fuer Astronomie (MPIA Heidelberg), National Astronomical Observatories of China, New Mexico State University, New York University, The Ohio State University, Penn State University, Shanghai Astronomical Observatory, United Kingdom Participation Group, University of Portsmouth, University of Utah, University of Wisconsin, and Yale University. This work was supported in part by a World Premier International Research Center Initiative (WPI Initiative), MEXT, Japan.

\section{References}

Bershady, M. A., Verheijen, M. A. W., Swaters, R. A., Andersen, D. R., Westfall, K. B., \& Martinsson, T. 2010, ApJ, 716, 198

Cappellari, M. et al. 2011, MNRAS, 413, 813

Conroy, C. \& van Dokkum, P. 2012, ApJ, 747, 69

Croom, S. M. et al. 2012, MNRAS, 421, 872

de Zeeuw, P. T. et al. 2002, MNRAS, 329, 513

Gunn, J. E. et al. 2006, AJ, 131, 2332

Maraston, C. 2005, MNRAS, 362, 799

Sánchez, S. F. et al. 2012, A\&A, 538, A8

Smee, S. A., Gunn, J. E., Uomoto, A., et al. 2013, AJ, 146, 32 